\title{
Prevalência e Preditores de Burnout em Bombeiros
}

\author{
Lúcia Petrucci de Melo \\ Pontifícia Universidade Católica do Rio Grande do Sul, \\ RS, Brasil.
}

\author{
Mary Sandra Carlotto \\ Universidade do Vale do Rio dos Sinos, \\ RS, Brasil.
}

Resumo: Compreendida como um fenômeno psicossocial, a Síndrome de Burnout ocorre como resposta ao estresse laboral crônico e reflete importante desafio da vida profissional no século XXI. Profissionais que lidam com situações de emergência, por entrarem em contato com eventos traumáticos, estão mais suscetíveis ao comprometimento de seu bem-estar físico e emocional. A Síndrome de Burnout apresenta-se como uma das possíveis consequências geradas por esta atividade. O estudo teve por objetivo identificar a prevalência de Burnout em bombeiros, bem como verificar a existência de associação entre Burnout e as variáveis sociodemográficas, laborais e estratégias de enfrentamento. A amostra, probabilística, constituiu-se de 132 participantes. Para a coleta de dados, utilizou-se Questionário Sociodemográfico-laboral, Questionário para Avaliação da Síndrome de Burnout (CESQT) e Escala de Coping no Trabalho (COPE). Os resultados identificaram uma prevalência de 3,0\% de profissionais no Perfil 1 de Burnout e 2,3\% no Perfil 2. A análise de regressão multivariada (stepwise method) apontou as estratégias de enfrentamento como principais variáveis preditoras de Burnout. Este estudo contribui para aprofundar as implicações de Burnout nesta categoria profissional, bem como ressaltar a relevância de estudos de intervenção voltados para o desenvolvimento de estratégias de enfrentamento como meio de promoção da saúde ocupacional destes profissionais.

Palavras-chave: Burnout, Coping, Bombeiros, Trabalho.

\section{Prevalence and Predictors of Burnout among Firefighters}

\begin{abstract}
Burnout is a psychosocial phenomenon that occurs as a response to chronic job stress and reflects a major challenge for the working life in the twenty-first century. By coming into contact with traumatic events, emergency professionals are more susceptible to compromise their physical and emotional well-being. Burnout presents itself as one of the possible consequences generated by this activity. The present study aimed to identify the prevalence of Burnout among firefighters as well as to verify the existence of an association among Burnout, sociodemographic and labor variables, and coping strategies. A probabilistic sample, consisted of 132 participants, was investigated using the Socio-demographic-labor Questionnaire, the Spanish Burnout Inventory (SBI) and the Coping at Work Scale (COPE). The results identified a prevalence of $3 \%$ of the professionals in the Burnout Profile 1, and $2.3 \%$ in the Burnout Profile 2. Multiple linear hierarchical regression analyses (stepwise method) showed that coping strategies seem to be predictors of Burnout among firefighters. This study contributes to deepen the implications of Burnout among firefighters, and to underscore the relevance of intervention studies aimed to create development coping strategies as a means of promoting occupational health among these professionals.
\end{abstract}

Keywords: Burnout, Coping, Firefighters, Work. 


\title{
Prevalencia y Predictores de Burnout en Bomberos
}

\begin{abstract}
Resumen: Entendido como un fenómeno psicosocial, el burnout se produce en respuesta al estrés laboral crónico y refleja un importante desafío de la vida laboral en el siglo XXI. Los profesionales que trabajan en situaciones de emergencia, al entrar en contacto con acontecimientos traumáticos, son más susceptibles al deterioro de su bienestar físico y emocional. El burnout se presenta como una de las posibles consecuencias generadas por esta actividad. El estudio tuvo como objetivo identificar la prevalencia de burnout en bomberos y verificar la existencia de una asociación entre burnout, variables sociodemográficas y laborales, y estrategias de afrontamiento. Una muestra probabilística de 132 participantes fue investigada utilizando el Cuestionario Sociodemográfico-laboral, el Cuestionario para la Evaluación del Síndrome de Burnout en el Trabajo (CESQT) y la Escala de Afrontamiento en el Trabajo (COPE). Los resultados identificaron una prevalencia de $3 \%$ de los profesionales en el Perfil 1 y de 2,3\% en el Perfil 2. El análisis multivariado de regresión (stepwise method) mostró las estrategias de afrontamiento como predictores del burnout. Este estudio ayuda a profundizar las implicaciones del burnout y subrayar la importancia de los estudios de intervención destinados a la elaboración de estrategias de afrontamiento para promover la salud ocupacional del bombero.
\end{abstract}

Palabras clave: Burnout, Estrategias de afrontamiento, Bomberos, Trabajo.

A Síndrome de Burnout (SB), apesar de ser um fenômeno psicossocial de natureza, causas e consequências muito conhecidas e difundidas, ainda reflete um importante desafio na vida profissional no século XXI. Compreendida como um processo que envolve variáveis cognitivas-atitudinais (diminuição da realização pessoal no trabalho), variáveis emocionais (exaustão emocional) e variáveis atitudinais (despersonalização) (Gil-Monte, 2001), ela ocorre como uma resposta crônica aos estressores interpessoais vinculados às questões relacionadas ao trabalho, presentes especialmente quando, no ambiente laboral, existem excessiva pressão e conflitos, aliados à falta de recompensas emocionais e reconhecimento (Harrison, 1999; Maslach, 2003; Maslach, Schaufeli, \& Leiter, 2001). Refere-se à etapa final das constantes tentativas do sujeito de lidar com essas condições negativas, ou a falha de enfrentar com eficácia tais situações estressantes (Byrne, 1993; Cherniss, 1980).

A definição mais aceita e empregada na literatura internacional da SB é a de Maslach e Jackson (1981), que a considera como um fenômeno multidimensional, estruturado em três dimensões: 1) Exaustão emocional, entendida como a ausência de energia e sentimento de esgotamento dos recursos emocionais do sujeito, os quais podem somar-se à frustração e tensão com a percepção de que o sujeito já não tem energia e disposição para atendar seus clientes ou colegas de trabalho; 2) Despersonalização ou desumanização, caracterizada por sentimentos negativos e insensibilidade emocional para com as pessoas com as quais o sujeito trabalha, passando a tratá-las de maneira distante e impessoal; e 3) Baixa realização profissional, distinguida por uma tendência de o sujeito fazer avaliação negativa de seu desempenho no trabalho e apresentar insatisfação com seu desenvolvimento profissional.

Outro modelo teórico, construído a partir de estudos qualitativos, que explica a SB é o desenvolvido por Gil-Monte (2005). Para esse autor, Burnout constitui-se em quatro dimensões: 1) Ilusão pelo trabalho, definida como o desejo do indivíduo de alcançar suas metas e trabalho, sendo este uma fonte de realização pessoal; 2) Desgaste psíquico, caracterizado pelo esgotamento emocional e físico decorrente de precisar lidar diariamente, em sua atividade laboral, com pessoas que apresentam ou causam problemas; 3) Indolência, refere-se à presença de atitudes negativas de indiferença e distanciamento com seus clientes, apresentando insensibilidade diante dos problemas das pessoas que necessitam atender; e 4) Culpa, definida pelo surgimento de sentimentos de culpabilização por atitudes e comportamentos não condizentes com as normas internas e cobrança social acerca do papel profissional. 
Essas quatro dimensões estabelecem dois perfis claramente diferenciados: Perfil 1, constituído das dimensões Ilusão ao trabalho, Desgaste psíquico e Indolência, em que ocorre a deterioração cognitiva e afetiva, que aparecem, inicialmente, como respostas às fontes de estresse laboral crônico, mas não incapacitam o sujeito para o exercício de seu trabalho; e Perfil 2, que ocorre quando se soma a Culpa às demais dimensões, podendo gerar maior dano ao indivíduo e, por conseguinte, podendo apresentar sérios problemas na execução de seu trabalho (Gil-Monte, 2005).

Os profissionais que lidam com situações de emergência estão mais vulneráveis a enfrentar eventos traumáticos e, por consequência, estão mais expostos a condições que afetam seu bem-estar físico e emocional (Riolli, \& Savicki, 2012). Estressores ocupacionais, quando persistentes, são considerados importantes preditores dos resultados de saúde mental entre esses profissionais, e a SB apresenta-se como uma das possíveis consequências geradas por esta atividade (Meyer et al., 2012). No caso dos profissionais bombeiros, os estudos apresentam como fatores estressores dessa profissão aspectos relacionados diretamente à natureza de suas atividades laborais, como o contato direto com a morte, acidentes envolvendo colegas de trabalho, necessidade de autocontrole de suas respostas emocionais, permanência constante em estado de alerta e a prestação de ajuda a pessoas jovens com ferimentos sérios ou mesmo óbito; e, ainda, o nível educacional do profissional e aspectos relacionados às condições e estrutura organizacional, como tipo de cargo ocupado, tempo de experiência profissional, tempo de atuação na instituição, qualificação profissional, jornada de trabalho, sobrecarga de trabalho, horas extras e possuir mais de um vínculo empregatício (Baptista, Morais, Carmo, Souza, \& Cunha, 2005; Beaton, \& Murphy, 1993; Cardoso, 2004; Santos, \& Passos, 2010; Silva, Lima, \& Caixeta, 2010).

No Brasil, são poucos os estudos que investigam a SB em bombeiros. Nesse contexto, em pesquisa que avaliou a depressão, SB e qualidade de vida em 101 bombeiros de uma região do interior de São Paulo, os resultados apresentaram sintomatologia depressiva, sendo 13,86\% com nível leve, 3,96\% nível moderado e $1,0 \%$ com nível severo e associação direta entre a sintomatologia depressiva e as dimen- sões de exaustão emocional e despersonalização, do Burnout (Baptista et al., 2005).

Em estudo desenvolvido com enfermeiros-bombeiros, realizado na cidade do Rio de Janeiro, os achados identificaram a SB em $84,5 \%$ da amostra, avaliando as dimensões do Burnout em alta, média e baixa tendência. Na dimensão exaustão emocional, $89,7 \%$ da amostra apontou alta tendência, 78,9\% apareceu com alta tendência na dimensão despersonalização e 79,3\% com alta tendência na dimensão envolvimento pessoal no trabalho. O mesmo estudo também revelou maior prevalência de Burnout nos profissionais com até cinco anos de atividade e em profissionais que possuíam mais de um vínculo empregatício (Santos, \& Passos, 2010).

O trabalho com 54 profissionais de Corpo de Bombeiros de uma cidade do Alto Paraíba, no estado de Minas Gerais, que objetivou analisar as três dimensões de Burnout, identificou alta exaustão emocional e despersonalização e baixa realização pessoal nos participantes (Silva et al., 2010). Em trabalho realizado em Portugal, que objetivou identificar as emoções no ambiente de trabalho e sua relação com o Burnout, os resultados demonstraram que as emoções positivas vivenciadas no ambiente de trabalho parecem ser protetoras de Burnout, enquanto que as emoções negativas aumentam o risco de exaustão emocional e despersonalização (Vara, \& Queirós, 2010).

A pesquisa com 101 bombeiros franceses identificou uma relação positiva entre a exaustão emocional e as exigências do trabalho, e negativa com controle de trabalho. Da mesma forma, a despersonalização associou-se positivamente com as exigências do trabalho e negativamente com controle de trabalho (Lourel, Abdellaoui, Chevaleyre, Paltrier, \& Gana, 2008).

Os fatores estressores da atividade de bombeiro associados às variáveis como personalidade e estratégias de enfrentamento têm demonstrado forte influência no desenvolvimento da SB (Moreno-Jiménez, Natera, Muñoz, \& Benadero, 2006; Pañacoba Puente, Diáz Gutiérrez, Goiri Pueyo, \& Espeanza y Veja Lopéz, 2000). No campo de estudos de estresse no trabalho, há uma linha que enfatiza a importância das variáveis individuais como atenuadoras dos efeitos deste estresse, entendendo que estas são produtos do sujeito baseado na valoração que o mesmo atribui ao evento estressor, fazendo com que a avaliação que os sujeitos realizam sobre um mesmo acontecimento seja dife- 
rente. Uma destas variáveis individuais que mais têm recebido atenção são as estratégias de enfrentamento - coping (Pañacoba Puente et al., 2000).

Estudos têm relatado que as estratégias de enfrentamento (coping) funcionam como preditores e mediadores entre o estresse percebido e suas consequências, desse modo, agindo como um fator de proteção à SB (Almeida, 2013; Gil-Monte, 2005; Hernández, Olmedo, \& Ibáñez, 2004; Shin et al., 2014). O coping é entendido como um conjunto de respostas intencionais, cognitivas e comportamentais utilizado, pelo sujeito, com o propósito de lidar com demandas específicas oriundas de situações estressantes, que podem ser aprendidas, utilizadas e descartadas (Lazarus, \& Folkman, 1984).

No modelo proposto por Folkman e Lazarus (1980), o coping é dividido em dois: focalizado no problema e na emoção. O coping focalizado no problema constitui-se no esforço despendido pelo sujeito para atuar na situação que deu origem ao estresse, tentando mudá-la. A função desta estratégia é alterar o problema existente na relação entre a pessoa e o ambiente que está causando a tensão. O coping focalizado na emoção é compreendido como um esforço para regular o estado emocional que é associado ao estresse e reduzir a sensação física desagradável que é gerada. Esses esforços são dirigidos a um nível somático e/ou a um nível de sentimentos, tendo por consequência a alteração do estado emocional do indivíduo.

A fim de ampliar o entendimento sobre as possíveis variedades de coping e fazer algumas diferenciações, os autores Carver, Scheier e Weintraub (1989) incluíram nesta classificação o coping de evitação, que corresponde às tentativas do indivíduo de evitar o enfrentamento seja diretamente com o problema ou mesmo com as emoções associadas a este. Assim, Carver et al. (1989) detalharam quinze tipos de coping. coping ativo, processo de estabelecer passos para remover, atenuar ou melhorar os efeitos do estressor; planejamento, atividade de pensar sobre alternativas para lidar com um estressor por intermédio de estratégias de ação; supressão de atividades concomitantes, supressão de atividades que distraiam o sujeito do foco representado pelo estressor; coping moderado, significa esperar uma oportunidade apropriada para a ação, restringindo a impulsividade; busca de suporte social por razões instrumentais, procura por conselho, auxílio, ou informação sobre o estressor; busca de suporte social por razões emocionais, busca por apoio moral, compaixão ou entendimento; foco na expressão de emoções, foco na experiência de estresse, expressando sentimentos negativos; desligamento comportamental, abandono das tentativas para atingir metas nas quais o estressor interfira; desligamento mental, uso de atividades alternativas para afastar o problema da mente; reinterpretação positiva, reinterpretar uma situação negativa ou tensa em termos positivos; negação, recusa em acreditar na existência do estressor, ou agir como se esse não fosse real; aceitação, percepção do estressor como real e, aceitação do estressor como um fenômeno natural; religiosidade, tendência a voltar-se para a religião como forma de aliviar a tensão; humor, estratégia de fazer graça da situação estressora; uso de substâncias, desviar do problema por meio do uso de substâncias psicoativas que interfiram na capacidade de avaliação das situações.

Trabalhos empíricos têm desvelado o poder do coping como um fator protetor de saúde, evidenciando que as estratégias de enfrentamento utilizadas pelos indivíduos são importantes para a redução ou aumento dos níveis de Burnout (Anshel, 2000; McPherson, 2012). Thornton (1992) identificou associação entre coping de evitação e Burnout em estudo com trabalhadores de saúde mental. Chan e Hui (1995) corroboram esse achado, em pesquisa com professores chineses, ao demonstrarem uma associação positiva entre o uso de estratégias de evitação e o aumento dos níveis de Burnout. Em estudo longitudinal com trabalhadores sociais, Koeske, Kirk, \& Koeske (1993) verificou que a utilização de estratégias focalizadas no problema promove uma melhor capacidade de enfrentar as situações difíceis no trabalho.

No caso dos profissionais bombeiros, que lidam com demandas extraordinárias, as estratégias de enfrentamento empregadas são reflexos da natureza de seu trabalho e possuem a função de proteger o profissional de situações desagradáveis ou ameaçadoras. Por isso, o desenvolvimento de habilidades de manobras de enfrentamento é importante, pois auxilia o trabalhador a lidar melhor com os problemas e dificuldades existentes no ambiente laboral, dá suporte aos trabalhadores, proporcionando condições de vida dentro e fora da organização e, consequentemente, melhorando a quali- 
dade do cuidado prestado ao cliente/vítima (Beaton, Murphy, Johnson, Pike, \& Corneil, 1999; Moreno, Gil, Haddad, \& Vannuchi, 2011).

Desta forma, para influenciar o desenvolvimento ou não da SB, as estratégias de enfrentamento devem estar de acordo com a necessidade particular de cada indivíduo, bem como precisam ser empregados ajustes ambientais para a redução de eventos adversos ao âmbito organizacional e melhora da resposta do indivíduo ao ambiente de trabalho (Moreno et al., 2011). Assim, o presente estudo tem como objetivos identificar a prevalência da Síndrome de Burnout em profissionais bombeiros em uma cidade de grande porte do sul do Brasil, bem como verificar se existe associação entre a SB e as variáveis sociodemográficas, laborais e estratégias de enfrentamento.

\section{Método}

\section{Participantes}

Este estudo foi realizado em um Comando do Corpo de Bombeiros do estado do Rio Grande do Sul - Brasil. A partir de uma lista contendo o nome de todos os 336 profissionais da instituição, foram selecionados, aleatoriamente, 165 bombeiros para compor a amostra, considerando os seguintes critérios de inclusão: estar em atividade há mais de seis meses e não ter saído de férias nos últimos três meses. $\mathrm{O}$ cálculo utilizado para determinar o tamanho da amostra considerou uma prevalência estimada de $50,0 \%$ para a SB, valor determinado quando não há informação sobre a proporção de interesse (Agranonik, \& Hirakata, 2011), margem de erro amostral de 5,0\%, nível de confiança de 95\%, design effect de $0,5 \%$ e taxa de não resposta de $20,0 \%$. Um total de 132 profissionais responderam ao estudo (80,0\% de taxa de resposta global), havendo uma perda amostral de $20,0 \%$ por recusa, transferência, afastamento ou férias.

Entre os participantes do estudo, 90,9\% eram do sexo masculino $(\mathrm{n}=120)$, com idade média de 35,76 anos (DP = 10,68), com companheiro(a) fixo (a) (78,3\%), com filhos $(56,8 \%)$ e escolaridade em nível de ensino médio completo (47,7\%). O tempo médio de atuação dos profissionais como bombeiro é de $13,62$ anos ( $\mathrm{DP}=11,34)$, sendo a maioria soldado $(57,3 \%)$ atuando como combatente de fogo $(72,5 \%)$ e atendendo uma média de seis ocorrências semanalmente (DP $=4,5)$.

\section{Instrumentos}

Os dados foram coletados por meio de três instrumentos autoaplicáveis: 1) Questionário sociodemográfico (sexo, idade, possui companheiro fixo, filhos e escolaridade) e laboral (tempo de atuação como bombeiro, setor, posto militar, quantidade de ocorrências atendidas semanalmente e outros empregos), questões construídas de acordo com literatura sobre Burnout em bombeiros; 2) Cuestionario para la Evaluación del Síndrome de Quemarse por el Trabajo CESQT (Gil-Monte, 2005), adaptado para o Brasil por Gil-Monte, Carlotto e Câmara (2010), para a avaliação da Síndrome de Burnout. O instrumento apresenta 20 itens distribuídos em quatro subescalas: Ilusão pelo trabalho (cinco itens, alfa = 0,86 ), Desgaste psíquico (quatro itens, alfa $=0,85$ ), Indolência (seis itens, alfa $=0,72$ ) e Culpa (cinco itens, alfa $=0,81$ ). Os itens são avaliados mediante uma escala de frequência de cinco pontos, de 0 (Nunca) a 4 (Todos os dias); e 3) Cope Inventory (Carver et al., 1989), versão adaptada para o uso no Brasil por Mazon, Carlotto e Câmara (2008), para identificar as estratégias de enfrentamento utilizadas pelos profissionais. Este questionário totaliza 60 itens com sistema de pontuação de 1 (não costumo fazer isso nunca) a 4 (costumo fazer isso muito). A escala se constitui de 15 fatores (quatro itens para cada fator) que determinam um perfil de estratégias de enfrentamento: coping ativo, planejamento, supressão de atividades concomitantes, coping moderado, busca de suporte social por razões instrumentais, busca de suporte social por razões emocionais, reinterpretação positiva e crescimento, aceitação, retorno para a religiosidade, foco na expressão das emoções, negação, comportamento descomprometido, desengajamento mental, humor e uso de substâncias. Na escala original, a fidedignidade encontrada em relação à consistência interna variou de 0,62 a 0,92 nos 15 fatores, o que foi considerado satisfatório pelos autores. Os autores atribuem a baixa consistência interna ao fato de esta subescala ser diferente das demais por apresentar uma maior diversidade de ações possíveis (Carver et al., 1989). 


\section{Procedimentos}

Após obter autorização da instituição para a execução do estudo, contataram-se as chefias a fim de apresentar o objetivo da pesquisa e obter apoio na aplicação dos instrumentos. A coleta foi realizada pela primeira autora do estudo. Os procedimentos éticos seguiram a Resolução n ${ }^{\circ} 466 / 12$ do Conselho Nacional de Saúde (Brasil, 2012) referente à pesquisa com seres humanos, sob Protocolo $\mathrm{n}^{\circ}$ 512.521. A análise do banco de dados foi realizada no Pacote de Estatística para Ciências Sociais (SPSS - Statistical Package of Social Science, versão 17.0).

Para identificar os casos de Perfil 1 e Perfil 2, foram aplicados os critérios do manual do CESQT (Gil-Monte, 2011). Foram considerados Perfil 1 os participantes que apresentaram pontuações $\geq$ P90 (percentil 90) na pontuação média dos 15 itens que formam as subescalas de Ilusão pelo trabalho (invertida), Desgaste psíquico e Indolência, mas $<$ P90 na subescala de Culpa. No Perfil 2 foram incluídos os casos com pontuações $\geq \mathrm{P} 90$ na pontuação média dos 15 itens supracitados e também $\geq$ P90 na subescala de Culpa.

Para a análise das estratégias de enfrentamento, os 15 fatores foram agrupados em três dimensões de enfrentamento, assim como nos estudos de Dobreva-Martinova, Villeneuve, Strickland e Matheson (2002) e de Litman (2006): copingfocalizado no problema (coping ativo, planejamento, supressão de atividades concomitantes e busca de suporte social por razões instrumentais); coping centrado na emoção (coping moderado, busca de suporte social por razões emocionais, reinterpretação positiva e crescimento, aceitação, retorno para a religiosidade, foco na emoção e humor); e coping de evitação (negação, comportamento descomprometido, desengajamento mental e uso de substâncias).

Estatísticas descritivas foram realizadas para calcular as frequências, médias e desvios padrão. A força e a direção das relações entre as variáveis foram determinadas usando-se a medida do coeficiente de correlação de Pearson. Antes de realizar a análise de regressão linear hierárquica (método Stepwise), foram verificados os pressupostos de multicolinearidade, normalidade, linearidade, homoscedasticidade e independência dos resíduos e outliers, sem que se identificasse violação que contraindicasse sua utilização. A análise assumiu como variável dependente as dimensões da Síndrome de Burnout, e como variáveis independentes, as sociodemográficas, laborais e estratégias de enfrentamento. A seleção das variáveis preditoras assumiu o nível de significância de $\mathrm{p}<0,05$. Na análise de regressão, o poder do efeito foi obtido pelos coeficientes de regressão padronizados calculados para cada modelo final, de acordo com Field (2009).

\section{Resultados}

\section{Prevalência}

Na Tabela 1 estão elencadas as médias, desvios padrão e consistência interna das escalas de avaliação da SB e do Coping utilizados neste estudo. A Ilusão pelo trabalho foi a que apresentou média $(3,44)$ mais elevada entre as dimensões de Burnout, e a Indolência foi a dimensão de média mais baixa, 0,87 , considerando a escala de pontuação. Quanto às estratégias de enfrentamento, o coping focalizado no problema teve a média mais elevada $(2,93)$; e o coping de evitação foi a estratégia de média mais baixa, 1,43, de acordo com a escala de pontuação.

Considerando a pontuação total da escala, a porcentagem de participantes que indicaram altos níveis de Burnout para o Perfil 1 foi de 3,0\% $(\mathrm{n}=4), \mathrm{e} 2,3 \%(\mathrm{n}=3)$ apresentaram escores iguais ou superiores ao percentil 90 sobre a dimensão Culpa (Perfil 2) (Tabela 2).

\section{Preditores de Burnout}

Na Tabela 3, é evidenciada a associação entre as dimensões do CESQT, Coping e variáveis sociodemográficas e laborais, revelando que as estratégias de enfrentamento foram as que mais se associaram com as dimensões de Burnout. O coping focado no problema apresentou uma associação com as dimensões Ilusão pelo trabalho e Culpa, o coping focado na emoção associou-se positivamente com a dimensão Culpa, enquanto que o coping de evitação associou-se positivamente com as dimensões Desgaste psíquico e Culpa. Em relação às variáveis sociodemográficas e laborais, somente a variável setor apresentou associação com todas as dimensões de Burnout, sendo uma associação negativa com a dimensão Ilusão pelo trabalho e positiva com as demais dimensões. 
Psicologia: Ciência e Profissão Jul/Set. 2016 v. 36 n³, 668-681.

Tabela 1

Média, Desvio Padrão, Alfa de Cronbach das Dimensões do CESQT e Coping.

\begin{tabular}{lccc}
\hline Variável & M & DP & A \\
\hline Ilusão pelo trabalho & 3,44 & 0,64 & 0,85 \\
Desgaste psíquico & 1,23 & 0,89 & 0,80 \\
Indolência & 0,87 & 0,59 & 0,70 \\
Culpa & 0,98 & 0,71 & 0,83 \\
Coping focado no problema & 2,93 & 0,50 & 0,84 \\
Coping focado na emoção & 2,43 & 0,39 & 0,70 \\
Coping de evitação & 1,43 & 0,30 & 0 \\
\hline
\end{tabular}

CESQT: Questionário para Avaliação da Síndrome de Burnout.

Tabela 2

Porcentagem de profissionais bombeiros com nível crítico da SB de acordo com os Percentis (P) do Manual do CESQT.

\begin{tabular}{lcc}
\hline Variável & $\mathrm{P}<90$ & $\mathrm{P} \geq 90$ \\
\hline Ilusão pelo trabalho & $89(67,4 \%)$ & $43(32,6 \%)$ \\
Desgaste psíquico & $124(93,9 \%)$ & $8(6,1 \%)$ \\
Indolência & $118(89,4 \%)$ & $14(10,6 \%)$ \\
Culpa & $111(84,1 \%)$ & $21(15,9 \%)$ \\
Perfil 1 & $128(97,0 \%)$ & $4(3,0 \%)$ \\
Perfil 2 & $129(97,7 \%)$ & $3(2,3 \%)$ \\
\hline
\end{tabular}

SB: Síndrome de Burnout; CESQT: Questionário para Avaliação da Síndrome de Burnout.

Tabela 3

Matriz de correlação entre dimensões CESQT, Coping e variáveis sociodemográficas e laborais.

\begin{tabular}{lcccc}
\hline Variável & IL & DP & IN & CL \\
\hline Coping focalizado no problema & $0,326^{* *}$ & $-0,086$ & $-0,149$ & $0,181^{*}$ \\
Coping focalizado na emoção & 0,171 & 0,119 & $-0,310$ & $0,276^{* *}$ \\
Coping de evitação & $-0,168$ & $0,205^{*}$ & 0,153 & $0,349^{* *}$ \\
Idade & 0,040 & $-0,091$ & $-0,111$ & 0,105 \\
Possui companheiro(a) fixo(a)* & $-0,049$ & 0,146 & 0,107 & 0,056 \\
Possui filhos* & 0,112 & $-0,076$ & $-0,059$ & 0,008 \\
Número de filhos & 0,058 & 0,056 & 0,090 & 0,098 \\
Escolaridade & $-0,117$ & 0,171 & 0,153 & $-0,004$ \\
Tempo de atuação como bombeiro & 0,058 & $-0,072$ & $-0,089$ & 0,120 \\
Setor* & $-0,211^{*}$ & $0,255^{* *}$ & $0,221^{*}$ & $0,214^{*}$ \\
Posto militar & 0,128 & $-0,027$ & $-0,136$ & $-0,013$ \\
Número de ocorrências semanais & 0,152 & 0,055 & $-0,035$ & $-0,103$ \\
Número de ocorrências graves mensais & 0,034 & 0,018 & 0,041 & 0,120 \\
Executa outra atividade profissional & 0,117 & $-0,080$ & $-0,002$ & $-0,093$ \\
\hline
\end{tabular}

IL: Ilusão pelo trabalho; DP: Desgaste psíquico; IN: Indolência; CL: Culpa.

** $\mathrm{p}<0,01$.

${ }^{*} \mathrm{p}<0,05 .{ }^{\mathrm{a}} 0$ - Sim, 1 - Não.

e 0 - Combate, 1 -Administrativo. 
Na Tabela 4 são apresentados os resultados da análise de regressão multivariada hierárquica, considerando como variável dependente as dimensões de Burnoute como preditores o coping, as variáveis sociodemográficas e laborais. O resultado para Ilusão pelo trabalho identificou um modelo preditor composto pelo coping centrado no problema e número de ocorrências semanais, explicando $23,0 \%$ da variância para essa dimensão. Assim, o maior uso de estratégias de coping focado no problema $(\beta=0,417)$ e o maior número de ocorrências semanais atendidas $(\beta=0,254)$ predizem uma maior Ilusão pelo trabalho.

Em relação ao Desgaste psíquico, o modelo final foi explicado pelo coping de evitação e nível de escolaridade, com 11,0\% de sua variabilidade total. Ou seja, o maior uso de estratégias de coping de evitação ( $\beta=0,316)$ combinado ao maior nível de escolaridade $(\beta=0,256)$ prevê maior Desgaste psíquico dos profissionais bombeiros. A Indolência foi prevista pelo coping focalizado no problema e o coping de evitação. Este modelo explicou $18,0 \%$ da variância para essa dimensão. O menor uso de coping centrado no problema $(\beta=-0,412)$ combinado ao maior uso de coping de evitação $(\beta=0,251)$ previu níveis mais elevados de Indolência. A dimensão Culpa foi expli- cada pelo uso de estratégias de coping de evitação, com $24 \%$ da variância. O maior uso deste estilo de enfrentamento $(\beta=0,505)$ prevê maior Culpa. Os resultados revelam um poder de efeito predominantemente entre médio $\left(R^{2}=0,145\right)$ e elevado $\left(R^{2}=0,264\right)$, com exceção da variável coping evitação, que apresentou um poder considerado pequeno $\left(\mathrm{R}^{2}=0,080\right)$, de acordo com os parâmetros recomendados por Field (2009).

\section{Discussão}

O estudo teve por objetivo identificar a prevalência da Síndrome de Burnout em bombeiros e seus preditores sociodemográficos, laborais e estratégias de enfrentamento. Quanto à prevalência, foram identificados $3,0 \%(n=4)$ dos profissionais no Perfil 1 de Burnout, e 2,3\% ( $(=3)$ dos profissionais no Perfil 2. Os participantes que se enquadram no Perfil 2 são os que, segundo a legislação brasileira, podem ser considerados casos de Burnout. No Brasil, a SB é contemplada na Classificação Internacional das Doenças no 10 (CID-10), com o código Z-73.0, e no Decreto $n^{\circ}$ 3048, de 06 de maio de 1999, do Ministério da Previdência e Assistência Social do Brasil

Tabela 4

Modelo de regressão linear múltipla do CESQT, Coping e variáveis sociodemográficas e laborais.

\begin{tabular}{|c|c|c|c|c|c|c|c|}
\hline & $\mathrm{R}^{2}$ & $\mathrm{R}^{2 \text { Adjusted }}$ & $\mathrm{R}^{\text {change }}$ & B & SE & Beta & $\mathrm{t}$ \\
\hline \multicolumn{8}{|l|}{ Ilusão pelo trabalho } \\
\hline Coping focalizado no problema & 0,201 & 0,187 & 0,201 & 0,566 & 0,154 & $0,417^{* *}$ & 3,676 \\
\hline Número de ocorrências semanais & 0,264 & 0,239 & 0,064 & 0,037 & 0,016 & $0,254^{*}$ & 2,238 \\
\hline Modelo F & & & & $10,421^{* *}$ & & & \\
\hline \multicolumn{8}{|l|}{ Desgaste psíquico } \\
\hline Coping de evitação & 0,080 & 0,064 & 0,080 & 0,870 & 0,338 & $0,316^{*}$ & 2,578 \\
\hline Escolaridade & 0,145 & 0,115 & 0,065 & 0,323 & 0,154 & $0,256^{*}$ & 2,095 \\
\hline Modelo F & & & & $4,907^{*}$ & & & \\
\hline \multicolumn{8}{|l|}{ Indolência } \\
\hline Coping focalizado no problema & 0,145 & 0,130 & 0,145 & $-0,451$ & 0,129 & $-0,412^{* *}$ & $-3,497$ \\
\hline Coping de evitação & 0,207 & 0,180 & 0,062 & 0,440 & 0,206 & $0,251^{*}$ & 2,134 \\
\hline Modelo F & & & & $7,571^{* *}$ & & & \\
\hline \multicolumn{8}{|l|}{ Culpa } \\
\hline Coping de evitação & 0,255 & 0,242 & 0,255 & 1,113 & 0,248 & $0,505^{* *}$ & 4,490 \\
\hline Modelo F & & & & $20,164^{* *}$ & & & \\
\hline
\end{tabular}

** $\mathrm{p}<0,01$.

${ }^{*} \mathrm{p}<0,05$. 
(Diário Oficial da União 12.05.99 - no 89), que apresenta, na Lista B do Regulamento da Previdência, a Nova Lista de Doenças Profissionais e Relacionadas ao Trabalho, no entanto, para que o diagnóstico seja confirmado, seria necessário realizar entrevista clínica com os casos identificados.

A prevalência da SB pode variar conforme a natureza do trabalho, cultura e país em que o trabalhador está inserido. Estudos hispânicos que utilizaram o CESQT para estimar a prevalência de Burnout obtiveram percentuais de $11,7 \%$, para o Perfil $1 ; 1,3 \%$, para o Perfil 2 em profissionais que trabalham com pessoas com deficiência (Gil-Monte, 2005); e 47,15\%, para Perfil 1; e 3,25\%, para Perfil 2 em pediatras que atuam em hospital (Marucco, Gil-Monte, \& Flamenco, 2007).

A baixa prevalência de Burnoutidentificada nesta investigação faz pensar na Ilusão pelo trabalho como um possível fator protetor para essa categoria profissional visto que, se comparada com as demais dimensões, apresentou o maior percentil $(32,6 \%)$ e a média $(3,44)$ mais elevada, considerando a escala de pontuação. Estudos realizados com bombeiros, igualmente identificaram níveis elevados da dimensão Realização profissional (Gaspar \& Neves, 2014; Murta, 2013). Esse resultado pode ser entendido pelo fato de que, ainda que expostos a situações críticas e adversas, a atividade profissional dos bombeiros possui uma representação social de resgate e salvamento que, aliada a um perfil de profissional movido por um sentimento de altruísmo, pode contribuir para que a Ilusão funcione como um elemento de contenção a Síndrome de Burnout. Essa motivação somada à presença de fatores de ajuda a outras pessoas, segundo Harrison (1983), contribui para o sentimento de eficácia do indivíduo na realização de seus objetivos profissionais.

Em relação aos preditores, a análise indicou as estratégias de enfrentamento como a principal variável relacionada à Síndrome de Burnout, assim, corroborando os achados de outras pesquisas que objetivaram estimar o poder explicativo do coping sobre o desenvolvimento da SB (Mella, \& Bouti, 2013; Montero-Marin, Prado-Abril, Demarzo, Gascon, \& García-Campayo, 2014; Moura, 2007; Myendeki, 2008; Shin et al., 2014). A dimensão Ilusão pelo trabalho teve maior poder explicativo pelas variáveis coping centradas no problema e número de ocorrências semanais. Na medida em que há maior utilização de estratégias como coping ativo, planejamento, supressão de atividades concomitantes ou busca de suporte social por razões instrumentais e um maior número de ocorrências atendidas, aumenta o desejo de alcançar as metas e o trabalho passa a ser uma fonte de realização pessoal para o profissional.

Considerando que, para o exercício dessa profissão, há uma capacitação técnica específica e uma formação que exige acurácia e precisão na execução de suas atividades, esperava-se que as estratégias de enfrentamento centradas no problema estivessem entre as mais adotadas por tais profissionais como forma de obterem êxito em seus atendimentos. Essas estratégias têm se mostrado associadas com níveis mais baixos de Burnout, uma vez que a sua adoção aumenta a percepção de controle sobre os acontecimentos significativos, com isso, revelando-se um tipo de enfrentamento saudável e eficaz (Moura, 2007; Shin et al., 2014).

Quanto ao número de ocorrências atendidas predizer a dimensão Ilusão pelo trabalho, pode-se pensar na satisfação dos profissionais em relação à natureza do serviço prestado à comunidade. Esse perfil relacionado à necessidade de correr riscos não apenas físicos, mas também sociais, a fim de realizar-se, tem sido estudado como um traço de personalidade de procura de sensações (sensation seeking) frequentemente presente em pessoas que procuram profissões de exposição ao risco (Zuckerman, 1994). Assim, quanto maior a frequência com que prestam seus serviços à comunidade, maior a percepção de sua contribuição social, assim, aumentando seu sentimento de êxito profissional.

As variáveis coping de evitação (negação, comportamento descomprometido, desengajamento mental e uso de substâncias) e escolaridade tiveram maior poder explicativo para a dimensão Desgaste psíquico. A estratégia de enfrentamento de evitação gera implicações negativas para o bem-estar do indivíduo, aumentando seus níveis de estresse no trabalho (Dobreva-Martinova et al., 2002; Mostert, \& Joubert, 2005; Myendeki, 2008). Pode-se inferir para esse fenômeno que, na tentativa de o profissional controlar suas respostas emocionais frente à situação estressante, ele lança mão de comportamentos de fuga, no entanto, como o problema não é resolvido, será necessário despender um maior esforço emocional para manter o afastamento das pessoas com quem precisa lidar, ocasionando, consequentemente, maior desgaste emocional. 
Em relação à escolaridade, pelo plano de carreira da instituição, os profissionais com maior nível de instrução ocupam os postos de coordenação e chefia. Desta forma, pode-se pensar que a influência dessa variável para explicar o Desgaste psíquico deve-se ao fato de que a estes cabem as funções de gestão, sendo responsáveis pelas equipes ou respondendo pelo comando da instituição. Maslach (1978) refere que o papel desempenhado pelo trabalhador possui forte influência sobre o nível de estresse emocional do profissional. Com isso, na medida em que passa a ocupar uma posição de liderança e gestão, o profissional deixa de executar atividades de atendimento à comunidade; passando a envolver-se, predominantemente, com atividades administrativas e burocráticas da instituição, portanto, distanciando-se das principais atividades que motivaram sua escolha profissional.

Quanto à dimensão Indolência, ser explicada pela menor utilização de coping centrado no problema e maior utilização de coping de evitação, reforça a evidência, já identificada nas dimensões discutidas anteriormente, de que a utilização de estratégias orientadas ao problema são mais efetivas e saudáveis no enfrentamento de situações estressantes do que aquelas orientadas à emoção ou à evitação. Dessa forma, aqueles profissionais que conseguirem enfrentar diretamente o estressor experimentarão uma sensação de maior satisfação e dever cumprido para com a comunidade. Em contrapartida, os profissionais que evitarem esse contato acabarão por expressar comportamentos de indiferença e falta de sensibilidade, comprometendo sua relação com a comunidade atendida e intensificando sua Indolência.

A dimensão de Culpa foi explicada pela variável coping de evitação, sugerindo que o acúmulo de estressores gerados pelo distanciamento faz com que os bombeiros sintam-se mais responsáveis pelas atitudes negativas desenvolvidas em seu trabalho, especialmente com as pessoas com quem necessitam estabelecer relações. Visto que as exigências da sociedade causam impacto na vida das pessoas, essa explicação pode ser compreendida à luz da relação entre o trabalho executado e o esforço em adaptar-se às expectativas sociais (Murta, 2013). Na medida em que o profissional bombeiro utiliza estratégias de enfrentamento de evitação, ele acaba desenvolvendo atitudes negativas com a população, que são consi- deradas condutas inadequadas tanto pela instituição como pelos valores sociais, elevando, consequentemente, a Culpa.

\section{Considerações Finais}

A Organização Mundial da Saúde - OMS (Leka, \& Jain, 2010) considera Burnout um grave problema ocupacional devido às suas negativas implicações físicas e mentais. Desde seu surgimento, na década de 1970, os estudos sobre a SB têm se estendido para uma ampla variedade de profissões. No entanto, apesar de tratar-se de uma profissão exposta a elevado estresse ocupacional e a riscos físicos e psicológicos causados pela exposição a eventos traumáticos, as pesquisas envolvendo bombeiros ainda são pouco numerosas (McCall, \& Salama, 1999; Moreno Jiménez et al., 2006), desse modo, reforçando a importância de estudos que permitam aprofundar as implicações dessa temática no tocante a essa categoria profissional.

Assim, a maior contribuição deste estudo foi identificar a prevalência da SB em bombeiros e analisar seus principais preditores, destacando a importância das estratégias de enfrentamento como variável explicativa deste fenômeno e o sentimento de realização profissional como fator de contenção da Síndrome de Burnout. Deve-se destacar como forças do presente estudo os bons indicadores do poder do efeito obtidos em seus resultados.

Sugere-se, para a consolidação de seus resultados, que novos estudos sejam realizados, também com amostras probabilísticas, em regiões do país com maior abrangência populacional, para possibilitar a generalização de seus resultados em termos nacionais e, assim, contribuir para a identificação de possíveis diferenças culturais e regionais. Estudos longitudinais e de método qualitativo também devem ser considerados na medida em que ampliam o campo de conhecimento sobre a identificação dos principais estressores da profissão e permitem realizar um acompanhamento da incidência.

Entre as limitações deste estudo, deve-se salientar o "efeito do trabalhador sadio", que pode ter afetado a taxa de incidência de Burnout. Em razão de a perda amostral ser de $20,0 \%$, é possível que profissionais afetados pela SB não tenham respondido à pesquisa. Ainda, alguns artefatos de respostas, como desejabilidade social, devem ser considerados, pois o método de avaliação consistiu em instrumentos de autorrelato. 
Tento em vista que o presente estudo é de corte transversal, ressalta-se também a impossibilidade de estabelecer relações causais entre as variáveis investigadas.

Os achados sugerem que o profissional bombeiro pode se beneficiar de programas de treinamento

\section{Referências}

Agranonik, M., \& Hirakata, V. N. (2011). Cálculo de tamanho de amostra: proporções. Revista do Hospital de Clínicas de Porto Alegre, 31(3), 382-388. Recuperado de http://seer.ufrgs.br/index.php/hcpa/article/ view/23574/15837

Almeida, H. (2013). A longitudinal study about stress, burnout and coping. International Journal of Human Resource Studies, 3(2), 109-125. doi:10.5296/ijhrs.v3i2.4136

Anshel, M. H. (2000). A conceptual model and implications for coping with stressful events in police work. Criminal Justice and Behaviour, 27, 375-400. doi:10.1177/0093854800027003006

Baptista M. N., Morais, P. R., Carmo, N. C., Souza, G. O., \& Cunha, A. F. (2005). Avaliação de depressão, síndrome de burnout e qualidade de vida em bombeiros. Psicologia Argumento, 23(42), 47-54. Recuperado de http://www2.pucpr.br/reol/pb/index.php/ $\mathrm{pa}$ ?dd1 $=176 \& d d 99=$ view\&dd98=pb

Beaton, R., Murphy, S., Johnson, C., Pike, K., \& Corneil, W. (1999). Coping responses and posttraumatic stress symptomatology in urban fire service personnel. Journal of Traumatic Stress, 12(2), 293-308. doi:10.1023/a:1024776509667

Beaton, R. D., \& Murphy, S. A. (1993). Sources of occupational stress among firefighter/EMTs and firefighter/paramedics and correlations with job-related outcomes. Prehospital and Disaster Medicine, 8(2), 140-150. doi:10.1017/S1049023X00040218

Brasil. (2012). Ministério da Saúde, Conselho Nacional de Saúde. Resolução no 466, de 12 de dezembro de 2012. [Aprovar diretrizes e normas regulamentadoras de pesquisas envolvendo seres humanos]. Brasília, DF: Conselho Nacional de Saúde.

Byrne, B. M. (1993). The maslach burnout inventory: testing for factorial validity and invariance across elementary, intermediate and secondary teachers. Journal of Occupational and Organizational Psychology, 66(3), 197-212. doi:10.1111/j.2044-8325.1993.tb00532.x planejados para capacitá-lo quanto ao uso de estratégias de enfrentamento. Com isso, ressalta-se a importância de estudos de intervenção que investiguem, por meio de diferentes abordagens, a promoção da saúde desses profissionais.

Cardoso, L. A. (2004). Influências dos fatores organizacionais no estresse de profissionais bombeiros (Dissertação Mestrado não publicada). Universidade Federal de Santa Catarina, Florianópolis.

Carver, C. S., Scheier, M. F., \& Weintraub, J. K. (1989). Assessing coping strategies: a theoretically based approach. Journal of Personality and Social Psychology, 56(2), 267-283. doi:10.1037/0022-3514.56.2.267

Chan, D. W., \& Hui, E. K. P. (1995). Burnout and coping among Chinese secondary school teachers in Hong-Kong. British Journal of Educational Psychology, 65(1),15-25. doi:10.1111/j.2044-8279.1995.tb01128.x

Cherniss, C. (1980). Professional burnout in human service organizations. New York: Praeger.

Dobreva-Martinova, T., Villenueve, M., Strickland, L., \& Matheson, K. (2002). Occupational role stress in the Canadian forces: its association with individual and organizational well-being. Canadian Journal of Behavioural Science, 34(2), 111-121. doi:10.1037/h0087161

Field, A. (2009). Discovering statistics with SPSS (2nd ed.). London: Sage.

Folkman, S., \& Lazarus, R. S. (1980). An analysis of coping in a middle-aged community sample. Journal of Health and Social Behavior, 21(3), 219-239. Recuperado de https://www.jstor.org/stable/2136617?seq=l\#page_scan_tab_contents

Gaspar, P., \& Neves, M. C. (2014). Study on burnout syndrome within the portugues firefighters population. In: V. Duffy \& N. Lightner (Eds.), Advances in human aspects of healthcare (pp. 356-364). USA: AHFE Conference.

Gil-Monte, P. R. (2011). CESQT: cuestionario para la evaluación del síndrome de quemarse por el trabajo. Madrid:TEA.

Gil-Monte, P. R. (2001). El síndrome de quemarse por el trabajo (síndrome de burnout): aproximaciones teóricas para su explicación y recomendaciones para la intervención. Psicopediahoy.2001. Recuperado dehttp:// psicopediahoy.com/burnout-teoria-intervencion/ 
Gil-Monte, P. R. (2005). El síndrome de quemarse por el trabajo (Burnout). Una enfermedad laboral en la sociedad del bienestar. Madrid: Pirâmide.

Gil-Monte, P. R., Carlotto, M. S., \& Câmara, S. (2010). Validation of the brazilian version of the "Spanish Burnout Inventory” in teachers. Revista de Saúde Pública, 44(1), 140-147. doi:10.1590/S0034-89102010000100015

Harrison, W.D. (1983). A social competence model of burnout. In: B. A. Farber (Ed.), Stress and burnout in the human services professions (pp. 29-39). New York: Pergamon Press.

Harrison, B. J. (1999). Are you destined to burn out? Fund Raising Management, 30(3), 25-27.

Hernández, Z. G. L., Olmedo, C. E., \& Ibáñez, I. (2004). Estar quemado (burnout) y su relación con el afrontamiento. Internacional Journal of Clinical and Health Psychology, 4(2), 323-336. Recuperado de http:// www.aepc.es/ijchp/articulos_pdf/ijchp-110.pdf

Koeske, G. F., Kirk, S. A., Koeske R. D. (1993). Coping with job stress: which strategies work best? Journal of Occupational and Organizational Psychology, 66(4), 319-335. doi:10.1111/j.2044-8325.1993.tb00542.x

Lazarus, R. L., \& Folkman, S. (1984). Stress, appraisal and coping. New York: Springer.

Leka, S., \& Jain, A. (2012). Health impact of psychosocial hazards at work: an overview. Geneva: World Health Organization.

Litman, J. A. (2006). The COPE inventory: dimensionality and relationships with approach - and avoidance-motives and positive and negative traits. Personality and Individual Differences, 41(2), 273-284. doi:10.1016/j.paid.2005.11.032

Lourel, M., Abdellaoui, S., Chevaleyre, S., Paltrier, M., \& Gana, K. (2008). Relationships between psychological job demands, job control and burnout among firefighters. North American Journal of Psychology, 10(3), 489-496.

McCall, M., \& Salama, P. (1999). Selection, training and support of relief workers: an occupational health issue. British Medical Jounal, 318(7176), 113-116. doi:10.1136/bmj.318.7176.113

McPherson, A. V. (2012). College student life and financial stress: an examination of the relation among perception of control and coping styles on mental health functioning (Unpublished doctoral dissertation).North Carolina State University, Raleigh, NC.

Marucco, M. A., Gil-Monte, P. R., \& Flamenco, E. (2007). Síndrome de quemarse por el trabajo (burnout) en pediatras de hospitales generales, estudio comparativo de la prevalencia medida com el MBI-HSS y el CESQT. Información Psicológica, 91/92, 32-42.

Maslach, C. (2003). Job burnout: new directions in research and intervention. Current Directions in Psychological Science, 12(5), 189-192. doi:10.1111/1467-8721.01258

Maslach, C. (1978). The client role in staff burn-out. Journal of Social Issues, 34(4), 111-124. doi:10.1111/j.1540-4560.1978.tb00778.x

Maslach, C., \& Jackson, S. E. (1981). The measurement of experienced burnout. Journal of Organizational Behavior, 2(2), 99-113. doi:10.1002/job.4030020205

Maslach, C., Schaufeli, W. B., \& Leiter, M. P. (2001). Job burnout. Annual Review of Psychology, 52, 397-422. doi:10.1146/annurev.psych.52.1.397

Mazon, V., Carlotto, M. S., \& Câmara, S. G. (2008). Síndrome de Burnout e estratégias de enfrentamento em professores. Arquivos Brasileiros de Psicologia, 60(1), 55-66. Recuperado de http://pepsic.bvsalud.org/scielo.php? script=sci_abstract\&pid=S1809-52672008000100006\& lng=pt\&nrm=iso\&tlng=pt

Mella, D. B., \& Bouti, A. P. K. (2013). Burnout and coping strategies in male staff from national police in Valparaíso, Chile. Iranian Journal of Public Health, 42(9), 950-959. Recuperado de http://www.ncbi. nlm.nih.gov/pmc/articles/PMC4453895/

Meyer, E. C., Zimering, R., Daly, E., Knight, J., Kamholz, B.W., \& Gulliver, S. (2012). Predictors of posttraumatic stress disorder and other psychological symptoms in trauma-exposed firefighters. Psychological Services, 9(1), 1-15. doi:10.1037/a0026414

Montero-Marin, J., Prado-Abril, J., Demarzo, M. M. P., Gascon, S., \& García-Campayo, J. (2014). Coping with stress and types of burnout: explanatory power of different coping strategies. Plos One, 9(2), e89090. doi:10.1371/journal.pone.0089090

Moreno, F. N., Gil, G. P., Haddad, M. C. L., \& Vannuchi, M. T. O. (2011). Estratégias e intervenções no enfrentamento da síndrome de burnout. Revista de Enfermagem UFRJ, 19(1), 140-145.

Moreno-Jiménez, B., Natera, N. I. M., Muñoz, A. R., \& Benadero, M. E. M. (2006). La personalidad resistente como variable moduladora del síndrome de burnout en una muestra de bombeiros. Psicothema, 18(3), 413-418. Recuperado de http:/ /www. psicothema.com/psicothema.asp?id=3231 
Mostert, K., \& Joubert, A. F. (2005). Job stress, burnout and coping strategies in the south african police service. South African Journal of Economic and Management Sciences, 8(1), 39-53. Recuperado de http://www.sajems.org/index.php/sajems/ article/viewArticle/ 1282

Moura, A. C. B. (2007). Estratégias de coping e estilos de vida como mediadores entre o stresse e o burnout nos bombeiros do distrito de Setúbal (Dissertação de mestrado não publicada). Universidade do Algarve, Faro.

Murta, S. M. S. (2013). Síndrome de burnout e valores de vida nos bombeiros sapadores da câmara municipal de Coimbra (Dissertação Mestrado não publicada). Instituto Superior de Línguas e Administração de Leiria, Leiria.

Myendeki, A. N. (2008). Job estress, burnout and coping strategies $f$ south African police officers (Dissertação de mestrado não publicada). University of Fort Hare, Fort Hare.

Pañacoba Puente, C., Diáz Gutiérrez, L., Goiri Pueyo, E., \& Esperanza y Veja López, R. (2000). Estrategias de afrontamiento ante situaciones de estrés: un análisis comparativo entre bomberos con y sin experiência. Revista de Psicología del Trabajo y de las Organizaciones, 16(3), 341-356. Recuperado de http://www.copmadrid.org/webcopm/recursoDenegado.html

Riolli, L., \& Savicki, V. (2012). Firefighters' psychological and physical outcomes after exposure to traumatic stress: the moderating roles of hope and personality. Traumatology, 18(3), 7-15. doi:10.1177/1534765611435565

Santos, P. G., \& Passos, J. P. (2010). O estresse e a síndrome de burnout em enfermeiros bombeiros atuantes em unidades de pronto-atendimento (UPAS). Revista de Pesquisa: Cuidado é Fundamental Online, 2 (Ed. Supl.), 671-675. doi:10.9789/2175-5361.2010. v0i0.\%25p

Shin, H., Park, Y. M., Ying. J. Y., Kim, B., Noh, H., \& Lee, S. M. (2014). Relationships between coping strategies and burnout symptoms: a meta-analytic approach. Professional Psychology: Research and Practice, 45(1), 44-56. doi:10.1037/a0035220
Silva, L. C. F., Lima, F. B., \& Caixeta, R. P. (2010). Síndrome deburnout em profissionais do corpo de bombeiros. Mudanças-Psicologia da Saúde, 18(1-2), 91-100. doi:10.15603/2176-1019/mud.v18n1-2p91-100

Thornton, P. I. (1992). The relation of coping, appraisal and burnout in mental health workers. The Journal of Psychology, 126, 261-271. doi:10.1080/00223980.1992.10543360

Vara, N., \& Queirós, C. (2010). Emotions and Burnout experienced by firefighters. Poster apresentado no 4th International Seminar of Positive Occupational Health Psycho, Lisboa, Portugal.

Zuckerman, M. (1994). Behavioral expressions and biosocial bases of sensation seeking. New York: Cambridge University Press.

\section{Lúcia Petrucci de Melo}

Mestre pela Pontifícia Universidade Católica do Rio Grande do Sul, Porto Alegre - RS. Brasil.

E-mail: lulucitamelo@hotmail.com

\section{Mary Sandra Carlotto}

Doutora. Docente da Universidade do Vale do Rio dos Sinos, São Leopoldo - RS. Brasil.

E-mail: mscarlotto@pesquisador.cnpq.br

Endereço para envio de correspondência: Pontifícia Universidade Católica do Rio Grande do Sul, Faculdade de Psicologia.

Av. Ipiranga, 6681. Partenon. CEP: 90619-900.

Porto Alegre - RS. Brasil.

Recebido 18/11/2014

Reformulado 28/06/2015

Aprovado 02/08/2016

Received 11/18/2014

Reformulated 06/28/2015

Approved 08/02/2016

Recibido 18/11/2014

Reformulado 28/06/2015

Aceptado 02/08/2016 
Melo, L. P., \& Carlotto, M. S. (2016). Prevalência e Preditores de Burnout em Bombeiros.

Como citar: Melo, L. P., \& Carlotto, M. S.. (2016). Prevalência e preditores de Burnout em bombeiros. Psicologia: Ciência e Profissão, 36(3): 668-681. doi:10.1590/1982-3703001572014

How to cite: Melo, L. P., \& Carlotto, M. S.. (2016). Prevalence and predictors of Burnout among firefighters. Psicologia: Ciência e Profissão, 36(3): 668-681. doi:10.1590/1982-3703001572014

Cómo citar: Melo, L. P., \& Carlotto, M. S.. (2016). Prevalencia y predictores de Burnout en bomberos. Psicologia: Ciência e Profissão, 36(3): 668-681. doi:10.1590/1982-3703001572014 